Research Article

\title{
Experimental Research on Wind-Induced Flag-Swing Piezoelectric Energy Harvesters
}

\author{
Jianjun Liu $\left(\mathbb{D}\right.$, Xianghua Chen $\left(\mathbb{D}\right.$, Yujie Chen $\left(\mathbb{D}\right.$, Hong Zuo $\left(\mathbb{D}\right.$, and Qun Li ${ }^{(D)}$ \\ State Key Laboratory for Strength and Vibration of Mechanical Structures School of Aerospace Engineering, \\ Xi'an Jiaotong University, Xi'an, China \\ Correspondence should be addressed to Hong Zuo; zuohong@mail.xjtu.edu.cn and Qun Li; qunli@mail.xjtu.edu.cn
}

Received 30 June 2021; Accepted 30 August 2021; Published 7 October 2021

Academic Editor: Zeqi Lu

Copyright (c) 2021 Jianjun Liu et al. This is an open access article distributed under the Creative Commons Attribution License, which permits unrestricted use, distribution, and reproduction in any medium, provided the original work is properly cited.

Piezoelectric cantilever beams, which have simple structures and excellent mechanical/electrical coupling characteristics, are widely applied in energy harvesting. When the piezoelectric cantilever beam is in a wind field, we should consider not only the influence of the wind field on piezoelectric beam but also the electromechanical coupling effect on it. In this paper, we design and test a wind-induced flag-swing piezoelectric energy harvester $(\mathrm{PEH})$. The piezoelectric cantilever beam may vibrate in the wind field by affixing a flexible ribbon to the free end as the windward structure. To fulfill the goal of producing electricity, the flexible ribbon can swing the piezoelectric cantilever in a wind-induced unstable condition. The experimental findings demonstrate that the flag-swing PEH performs well in energy harvesting when the wind field is excited. When the wind speed is $15 \mathrm{~m} / \mathrm{s}$, the peak-topeak output AC voltage may reach $13.88 \mathrm{~V}$. In addition, the voltage at both ends of the closed-loop circuit's external resistance is examined. The maximum electric power of the PEH may reach $43.4 \mu \mathrm{W}$ with an external resistance of $650 \mathrm{k} \Omega$. After passing through the AC-DC conversion circuit, the flag-swing PEH has a steady DC voltage output of $1.67 \mathrm{~V}$. The proposed energy harvester transforms wind energy from a wind farm into electrical energy for supply to low-power electronic devices, allowing for the creation and use of green energy to efficiently address the issue of inadequate energy.

\section{Introduction}

With the widespread application of low-power microelectronic devices in fields such as electronic information, artificial intelligence, aerospace, civil engineering, the machinery industry, and the engineering field, scientific researchers have begun to focus more on the devices' longterm power supply. Traditional batteries are currently the most common power supply mode for low-power-consumption devices, but they have the disadvantages of limited energy, short lifespan, environmental pollution, and difficulty in replacing them in some environments. As a result, a new power supply technology must be proposed as soon as possible to address the aforementioned disadvantages [1-4]. With the intellectualization, miniaturization, and low power consumption of electronic devices, the amount of electricity required by these devices will be lower and lower. Milliwatts or even microwatts of electrical power can meet the demand for energy, which can be extracted from the mechanical energy of functional materials in the vibrating surrounding environment. Because of their excellent mechanical and electrical coupling characteristics, piezoelectric materials are ideal for manufacturing a piezoelectric device that converts the mechanical energy contained in the environment into electrical energy. This piezoelectric device is called piezoelectric energy harvester (PEH) [5-7]. Wind energy, water energy, and tides in nature contain abundant mechanical energy, in which wind energy is an inexhaustible green and renewable resource. In the fields of collection and capturing vibration energy, wind-induced vibration has become a research focus of experts and scholars at home and abroad for its advantages of being ubiquitous and easy to collect [8-10]. When it flows through the surface of the object in the wind field, the wind load will generate aerodynamic loads on the object structure and the object structure will vibrate under the wind-induced excitation, such as vortex-induced resonance, galloping vibration, flutter, and chattering vibration. Among them, experts and scholars mainly focus on 
how to design, optimize, and improve the energy conversion from wind-induced mechanical energy to electrical energy using piezoelectric energy harvesters under the excitation of the wind field.

The related study of PEH has drawn more academicians' and researchers' attention since British scholars Yate and Williams [11] proposed the piezoelectric conversion method to capture ambient vibration energy in 1996. In 2001, Taylor et al. $[12,13]$ designed a PEH driven by river water flow to supply power for wireless sensor network nodes. The mechanism of this $\mathrm{PEH}$ is to utilize the river water flow to drive the piezoelectric material PVDF core component swinging, which converts the kinetic energy of water flows into electrical energy. When the speed of the water flow is $0.5 \mathrm{~m} / \mathrm{s}$, the output voltage of the piezoelectric sheet can reach $2 \mathrm{~V}$. The working environment of this device is in water, while this piezoelectric power generation is also suitable for the airflow environment. Priya et al. [14] designed a PZT piezoelectric windmill structure in 2005, which consists of a fan impeller, a transmission device, and a cantilever beam. When the wind blows the fan to rotate, the rotation of the fan is converted into the vibration of the cantilever beam by the transmission device. Thus, the PZT on the cantilever beam can harvest electricity, which achieves the purpose of energy harvesting. Li and Lipson [15] in 2009 investigated the practical application of energy recovery of piezoelectric cantilever beams under airflow conditions and designed a piezoelectric power generation device to harvest wind energy. The device's many blades swing back and forth in response to the wind vortex force, which also causes the cantilever beam to vibrate back and forth, generating electric energy. When the wind speed is $8 \mathrm{~m} / \mathrm{s}$ and the blades and piezoelectric sheets are reasonably distributed, the highest power generated by each blade can reach $296 \mu \mathrm{W}$. Since the piezoelectric coefficient of PVDF is much smaller than that of piezoelectric ceramic sheet PZT, Clair et al. [16] from Clemson University proposed an airflow-induced self-excited electrical device based on PZT ceramic in the same year. When the wind speed is between $7.5 \mathrm{~m} / \mathrm{s}$ and $12.5 \mathrm{~m} / \mathrm{s}$, the PZT ceramics will generate $0.1 \mathrm{~mW} \sim 0.8 \mathrm{~mW}$ of power under an external load of $49.65 \mathrm{k} \Omega$.

In 2011, Li et al. [17] used a piezo-leaf architecture to collect wind energy. The study found that when the wind direction is perpendicular to the piezo-leaf, the maximum electric power output is $600 \mu \mathrm{W}$ at a wind speed of $7 \mathrm{~m} / \mathrm{s}$ and the power density is $36 \mu \mathrm{W} / \mathrm{cm}^{3}$. In the same year, Weinstein et al. [18] proposed a vortex-shedding device to harvest energy from the airflow in the building's air-conditioning exhaust system. The device gets the maximum output power under the matched load and the wind speed is $3.2 \mathrm{~m} / \mathrm{s}$, and the power mean-square value reaches $510 \mathrm{~W}$. Liu et al. [19] designed a piezoelectric tree energy-harvesting device with hinges connecting a triangle at the end of a piezoelectric cantilever beam. The AC peak-to-peak voltage of $82 \mathrm{~V}$ can be obtained with the flutter wind speed in the wind field. In the closed circuit, when the external resistance is $680 \mathrm{k} \Omega$, it can have a DC output of $25.8 \mathrm{~V}$ and an electric power of $0.54 \mathrm{~mW}$ can be obtained.
Based on the above research, a wind-induced flag-swing $\mathrm{PEH}$ is proposed by pasting a flexible ribbon at the end of the piezoelectric cantilever beam in this paper, which makes full use of the mechanical energy contained in the wind field. The wind tunnel experiments were conducted to test and verify the reliability of the power supply performance of the flagswing $\mathrm{PEH}$.

\section{Mechanism of the Flag-Swing PEH in the Wind Field}

Inspired by a fluttering ribbon in the wind, this paper designed and investigated the characteristics of a flexible wind-induced flag-swing piezoelectric energy harvester to transform wind energy into electric energy, aiming to supply long-lasting power for the low-power-consumption smart electronic devices. The flexible ribbon in the unstable state stimulated by the wind field swings, and the excitation force generated by the wind field also drives the piezoelectric cantilever beam to swing left and right. At the same time, the piezoelectric material MFC senses the strain produced by the vibration of the cantilever beam and generates an instantaneous AC voltage based on the positive piezoelectric effect.

Firstly, the vibration resonance frequency of the piezoelectric cantilever beam energy harvester system is analyzed by utilizing the vibration scanning platform. Then, the vibration frequency of the energy harvester is optimally designed to reach the natural vibration frequency of the system so that the maximum vibration amplitude and the maximum output voltage can be obtained. Next, the flagswing $\mathrm{PEH}$ and the load resistor are connected to form a closed loop. Under the wind field, the flag-swing PEH can generate electricity continually, and the current can be detected at both ends of the resistor. The AC voltage generated by the flag-swing $\mathrm{PEH}$ is converted with an AC-DC conversion circuit that can provide power for low-powerconsumption electronic devices. This is the working principle of the flag-swing $\mathrm{PEH}$ in this article.

Due to the exceptional performance, adaptability, and reliability in a cost-effective instrument, MFC [20] was created by NASA Langley Research Center in 1999 and is widely utilized in the fields of low-profile actuators, sensors, and energy harvesters. In polymer matrices such as epoxy resin or polyimide, piezoelectrical fibers and an interdigital electrode are inserted to generate an MFC. It is extremely flexible and trustworthy for electronic equipment if large deformations are involved. It can be utilized as an energy harvester to convert wind power to electricity because of its good characteristics [21, 22]. The MFC-P2-2814 is the main material for this piezoelectric power harvester with a windinduced flag swing to study the energy-harvesting behavior. The flexible piezoelectric composite material MFC-2814-P2 is pasted with epoxy glue on the fixed end of the cantilever beam, and the flexible ribbon is pasted on the other end of the cantilever beam. The schematic diagram of the windinduced flag-swing $\mathrm{PEH}$ designed in this paper is shown in Figure 1. The cantilever beam selected for the experimental investigation is an epoxy composite plate with a size of $200 \mathrm{~mm}, 30 \mathrm{~mm}$, and $0.5 \mathrm{~mm}$. Young's modulus of the epoxy 


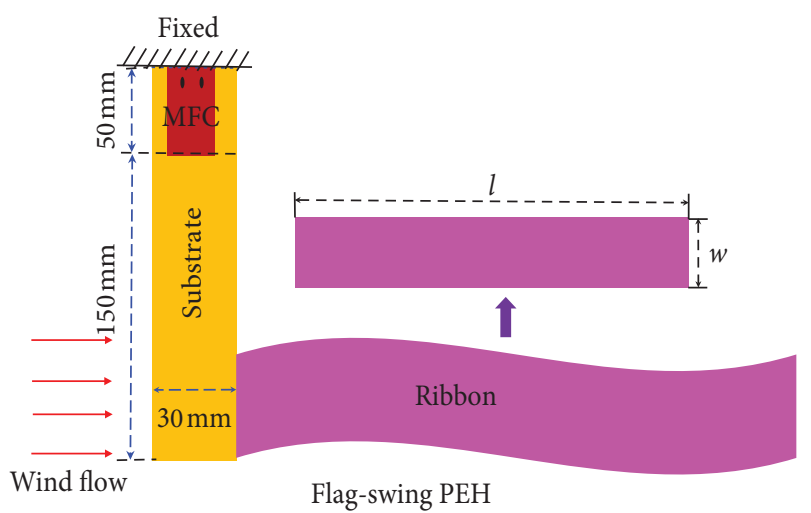

Figure 1: Schematic design of the wind-induced flag-swing PEH.

resin composite board is $3.5 \mathrm{GPa}$, of which Poisson's ratio is 0.32 and the density is $1960 \mathrm{~kg} / \mathrm{m}^{3}$. The size of the flexible silk ribbon is $500 \mathrm{~mm}, 5 \mathrm{~mm}$, and $0.2 \mathrm{~mm}$.

\section{Experiments and Analysis}

The vibration characteristics of the piezoelectric cantilever beam at different frequencies are tested and analyzed through the vibration scanning platform to obtain the vibration features and natural frequency of the $\mathrm{PEH}$ in this paper. The experimental equipment to test the vibration frequency is shown in Figure 2. At first, the piezoelectric cantilever beam energy harvester is installed and fixed on the vibration excitation platform, and the oscilloscope is connected to collect the open-circuit instantaneous voltage. The oscilloscope employed in this experiment for the electrical measurement is the Tektronix DPO-2012B type, and the input impedance of the oscilloscope probe is $1 \mathrm{M} \Omega$. The voltage drop from the MFC is small and almost negligible because the input impedance of the oscilloscope probe is much higher than the internal impedance of the MFC, which ranges from $10 \mathrm{k} \Omega$ to $50 \mathrm{k} \Omega$. Therefore, the electrical measurements by the oscilloscope can be regarded as precise. The fixed vibration frequency of the vibration platform provided by the signal generator is $1 \mathrm{~Hz}$, the vibration amplitude is $1 \mathrm{~V}$, and the vibration frequency output by the vibration platform is controlled between 0 and $120 \mathrm{~Hz}$. At this time, the vibration sweep frequency analysis of the piezoelectric cantilever beam energy harvester is conducted, and the open-circuit instantaneous voltage value is collected to analyze the vibration frequency response characteristics of the piezoelectric cantilever beam energy harvester.

The open-circuit instantaneous voltage results of the piezoelectric cantilever beam energy harvester obtained by the vibration scanning system are plotted in Figure 3. It is observed from the curve of the open-circuit voltage frequency response characteristics that there are three vibration modes of the piezoelectric cantilever beam, among which the first three natural frequencies $f_{1}, f_{2}$, and $f_{3}$ are $7.9 \mathrm{~Hz}, 38.0 \mathrm{~Hz}$, and $108.5 \mathrm{~Hz}$, respectively. The maximum peak-to-peak value of the instantaneous $\mathrm{AC}$ voltage output at the three natural resonance frequencies is $9.92 \mathrm{~V}, 25.68 \mathrm{~V}$, and $20.22 \mathrm{~V}$, respectively. According to the sweep frequency

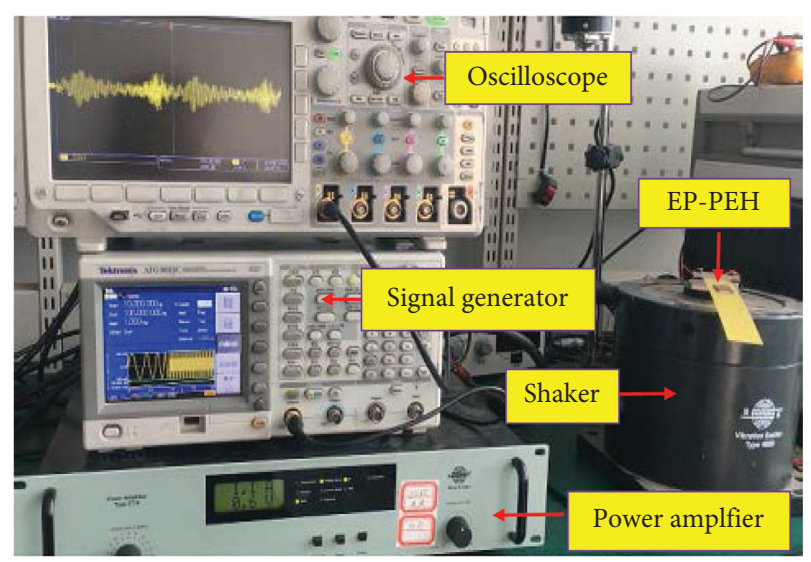

FIgURE 2: Experimental equipment of the vibration scanning system.

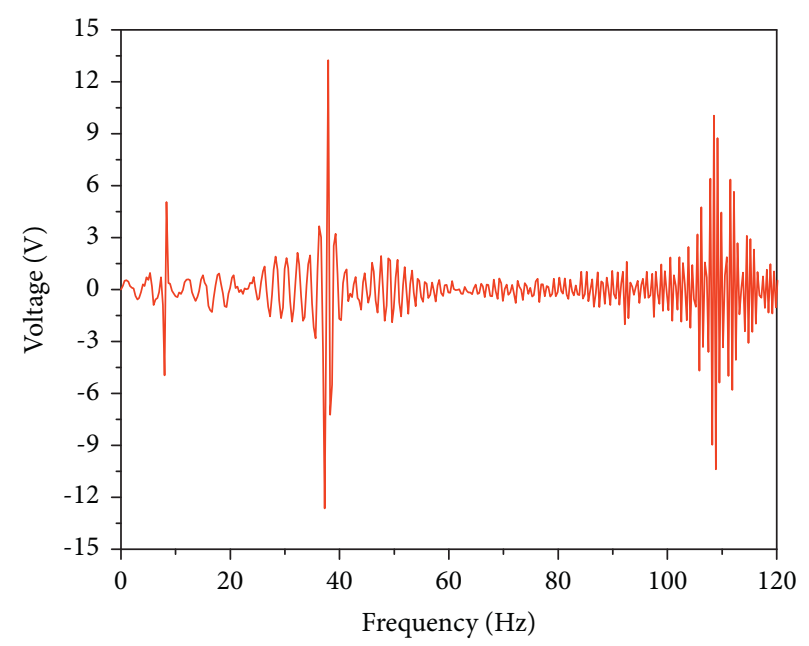

FIGURE 3: Curve of the open-circuit voltage frequency response characteristics generated by the piezoelectric cantilever beam energy harvester.

vibration results, when the vibration is at the mode $f_{2}=38 \mathrm{~Hz}$, the largest instantaneous peak-to-peak AC voltage is generated, where the bandwidth at the third-order vibration frequency is the largest.

After pasting a flexible ribbon at the end of the piezoelectric cantilever beam, the flag-swing $\mathrm{PEH}$ is tested in a wind tunnel, and the experimental equipment is shown in Figure 4 . When the wind speed in the wind field is $10 \mathrm{~m} / \mathrm{s}$, the vibration displacement of the flag-swing PEH measured with the laser displacement meter and the instantaneous output AC voltage waveform measured with the oscilloscope are as shown in Figures 5(a) and 5(b), respectively. The flexible ribbon pasted at the end of the piezoelectric cantilever can drive the piezoelectric cantilever to swing in the wind field so that the piezoelectric cantilever beam vibration can generate power, which can be found from the vibration curve in Figure 5(a). The waveform diagram in Figure 5(b) shows that the instantaneous AC voltage will be generated when the piezoelectric energy harvester swings from side to side and the maximum peak-to-peak AC voltage is $12.3 \mathrm{~V}$. 


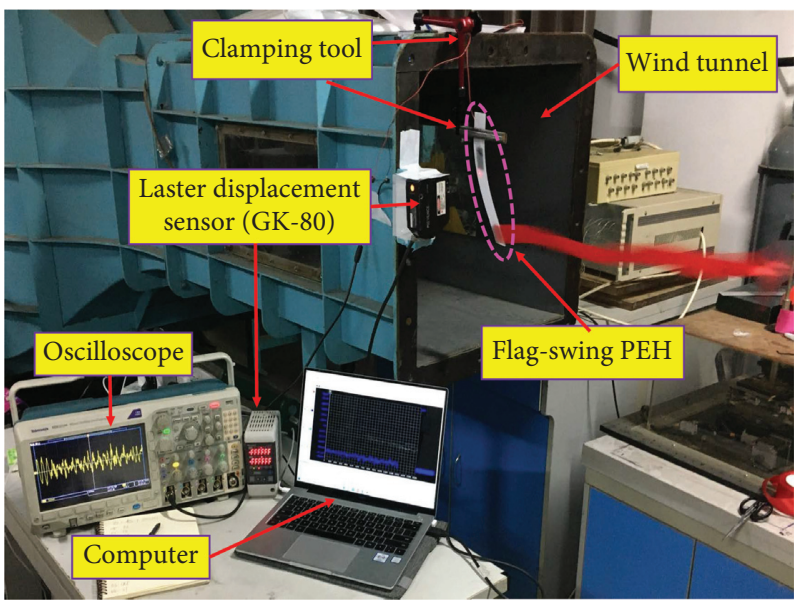

FIGURE 4: Experimental equipment of the wind tunnel test.

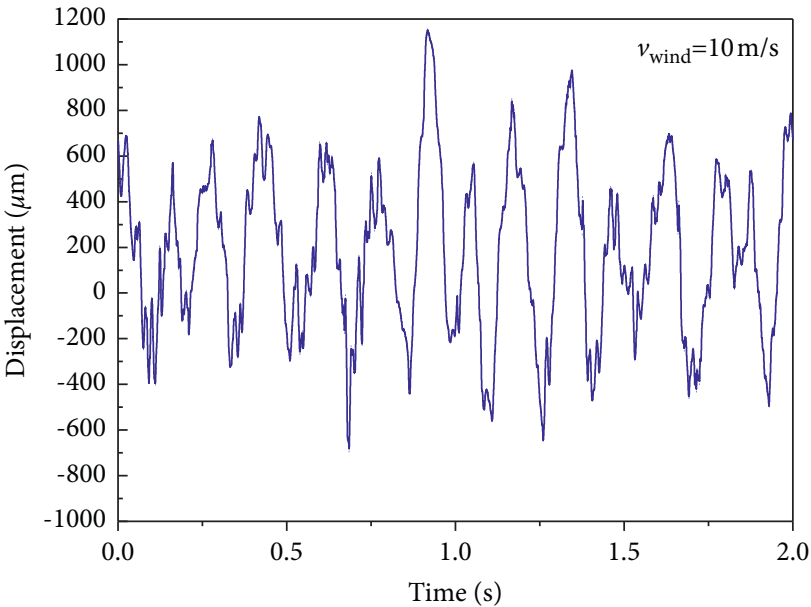

(a)

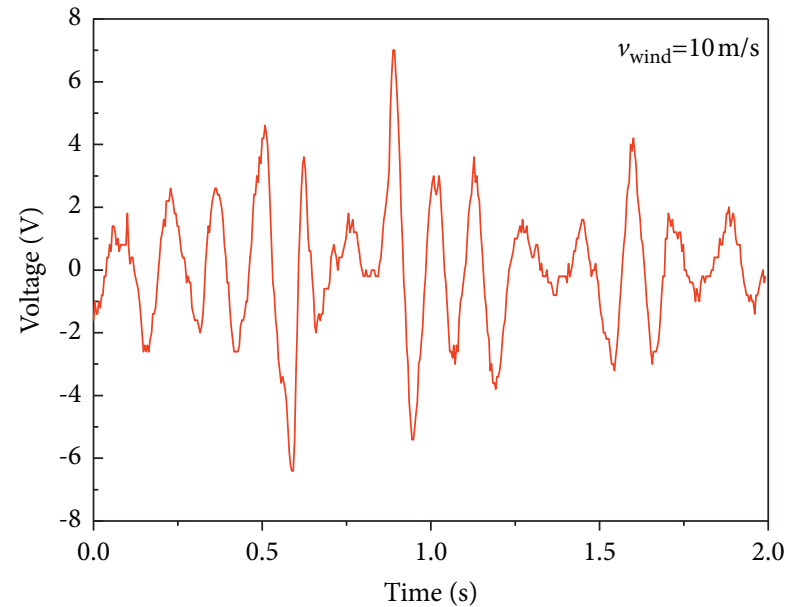

(b)

FIgURE 5: (a) The vibration displacement and (b) waveform of AC voltage generated by the piezoelectric energy harvester under a wind velocity of $10 \mathrm{~m} / \mathrm{s}$.

The wind speed in the wind field is not always stable. The performance of the piezoelectric energy harvester was investigated under a wind speed of $3 \mathrm{~m} / \mathrm{s} \sim 15 \mathrm{~m} / \mathrm{s}$ by adjusting the speed of the fan in the wind tunnel. The blue line with a circular marker represents the vibration frequency of the piezoelectric energy harvester under different wind speeds, as shown in Figure 6. According to the data, the vibration frequency of the harvester fluctuates between $10.95 \mathrm{~Hz}$ and $12.90 \mathrm{~Hz}$. The different vibration frequencies of the piezoelectric cantilever beam under different wind speeds result from the fluid-solid coupling between the flow field and the flexible ribbon. The red line with a triangle marker represents the AC voltage peak-to-peak value output by the energy harvester under different wind speeds. The output voltage of the energy harvester is affected by the vibration frequency of the piezoelectric cantilever and the excitation force of the flexible ribbon on the piezoelectric cantilever. The excitation force on the piezoelectric cantilever increases with the growth of wind speed. It can be found from the data results that the output voltage peak-to-peak value increases with the growth of wind speed, while the increment appears to be waveform. It is the influence of the wind field on the excitation frequency of the energy harvester. When the excitation frequency is the first-order natural vibration frequency of the energy harvester, the vibration amplitude will increase significantly and the corresponding output AC voltage also increases remarkably.

The frequency of the piezoelectric energy harvester is between $10.95 \mathrm{~Hz}$ and $12.90 \mathrm{~Hz}$ under different wind speeds, which differs by $3 \mathrm{~Hz} \sim 5 \mathrm{~Hz}$ from the first-order natural vibration frequency $f_{1}=7.9 \mathrm{~Hz}$ of the energy harvester measured by the excitation platform (shown in Figure 3 ) and by $25 \mathrm{~Hz} \sim 27 \mathrm{~Hz}$ from the second-order natural vibration frequency $f_{2}=38.0 \mathrm{~Hz}$ of the energy harvester. The excitation frequency of the wind field is not within the resonance frequency range of the energy-harvesting system. To make 


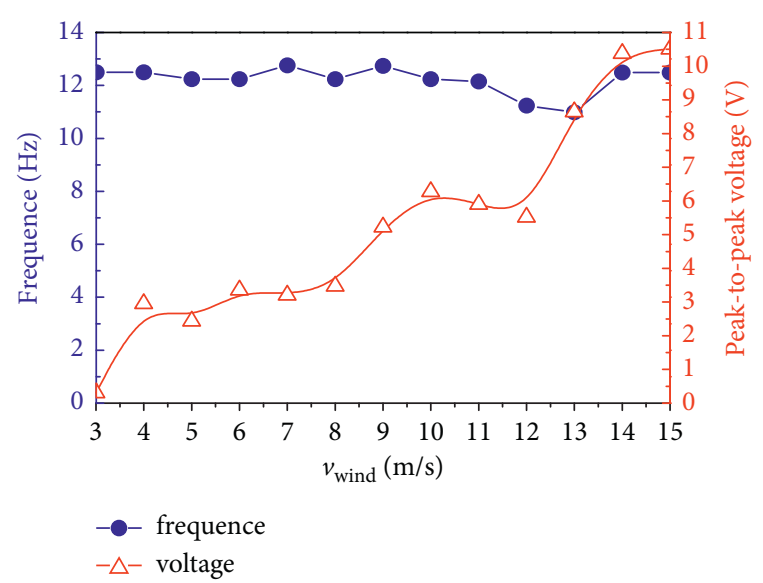

FIGURE 6: Frequency change incited by wind load and the value of peak-to-peak voltage under different wind velocities.

the vibration frequency of the piezoelectric energy harvester within the range of resonance frequency and reach the maximum vibration amplitude, the piezoelectric energy harvester should be further optimized to make the natural vibration frequency approach the excitation frequency of the wind field. Although a large AC voltage is generated by the flag-swing $\mathrm{PEH}$ at the second-order natural vibration frequency, it is easier to approach $f_{1}$ by optimizing the design of the flag-swing PEH. A metal block with a mass $m$ is added to the end of the piezoelectric cantilever beam (as shown in Figure 7), which is to reduce the first-order natural vibration frequency of the flag-swing $\mathrm{PEH}$. The equivalent mass of the PEH system is the sum of the original system's mass $m_{0}$ and the mass after the mass block $m$ has been added. The vibration frequency stimulated by the wind field is close to the first-order natural vibration frequency of the flag-swing PEH with an added metal block.

The energy-harvesting performance of the further-optimized flag-swing PEH is investigated in the wind field. When the wind speed is $10 \mathrm{~m} / \mathrm{s}$, the vibration displacement amplitude and the output instantaneous AC voltage of the energy harvester with and without a metal block are as shown in Figures 8(a) and 8(b). It can be observed from Figure 8(a) that when adding the metal block, the vibration displacement amplitude significantly increases and the curve fluctuates regularly. From the perspective of the vibration period, the vibration frequency of the energy harvester with a metal block excited by the wind field is significantly reduced. Figure $8(\mathrm{~b})$ compares the output AC voltages of the energy harvester before and after the metal block is added. After the metal block is added, the vibration frequency of the energy harvester decreases significantly, and in the meantime, the amplitude of the output AC voltage has been increased slightly.

When the wind speed is $3 \mathrm{~m} / \mathrm{s} \sim 15 \mathrm{~m} / \mathrm{s}$, the frequency change and output AC voltage peak-to-peak value of the optimized flag-swing PEH are shown in Figure 9, in which the blue line with a circle marker displays the frequency and the red line with a triangle marker displays the output AC voltage peak-to-peak value (the hollow marker represents without adding a metal block, and the solid marker

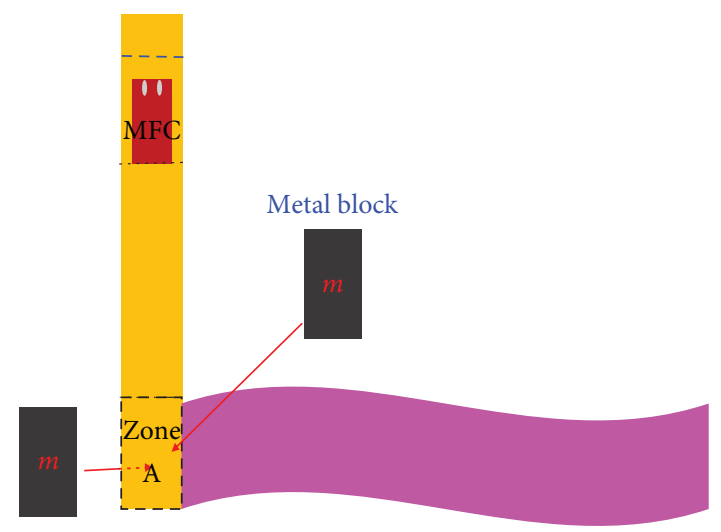

FIgURE 7: Schematic diagram of the flag-swing piezoelectric energy harvester with a metal block added at the end of piezoelectric beam.

represents adding a metal block). It can be observed from Figure 9 that after optimization, the vibration frequency of the flag-swing $\mathrm{PEH}$ incited by the wind field decreases significantly, while the output AC voltage peak-to-peak value increases greatly. The output $\mathrm{AC}$ voltage peak-to-peak value increases in a wave trend with the growth of wind speed, which is similar to the analysis shown in Figure 6 . When the wind speed intensity is $15 \mathrm{~m} / \mathrm{s}$, the AC voltage peak-to-peak value output by the energy harvester can reach $13.88 \mathrm{~V}$. The analysis indicates that the optimization method of adding a metal block at the end of the cantilever significantly improves the energy-harvesting performance. The main reason is that after a metal block is added, the vibration frequency of the energy harvester excited by the wind field approaches the first-order natural vibration frequency of the optimized energy harvester system, which enhances the swing amplitude of the energy harvester device, and thus a large AC voltage value is output.

A suitable external resistor in the closed-loop circuit should be selected for the flag-swing PEH to obtain the optimal electrical power output. The flag-swing PEH is connected with an external resistor $R$ to form a closed circuit, as shown in Figure 10. The value of the external resistance selected in the experiment is $10 \mathrm{k} \Omega \sim 3 \mathrm{M} \Omega$. The $V_{\mathrm{RMS}}$ at both ends of the resistance $R$ measured in the experiments and the different resistance values calculated by the electric power $P_{R}$ are shown in Figure 11, in which the blue line represents the root-mean-square voltage measured at both ends of the resistance $R$. The root-mean-square voltage increases with the growth of the resistance. When the external resistance $R=3.0 \mathrm{M} \Omega, U_{R}=8.72 \mathrm{~V}$. Under the same wind speed, the total voltage generated by the energy harvester is constant, and the larger the external resistance is, the larger the divided voltage will be. The relationship between electric power $P$ and resistance is

$$
P_{R}=I_{R}^{2} R=\left(\frac{V_{\mathrm{RMS}}}{R+r}\right)^{2} R=\frac{R}{(R+r)^{2}} V_{\mathrm{RMS}}^{2}
$$

where $r$ is the internal resistance of the energy harvester. $V_{\text {RMS }}$ denotes the root-mean-square voltage, and it can be expressed as 


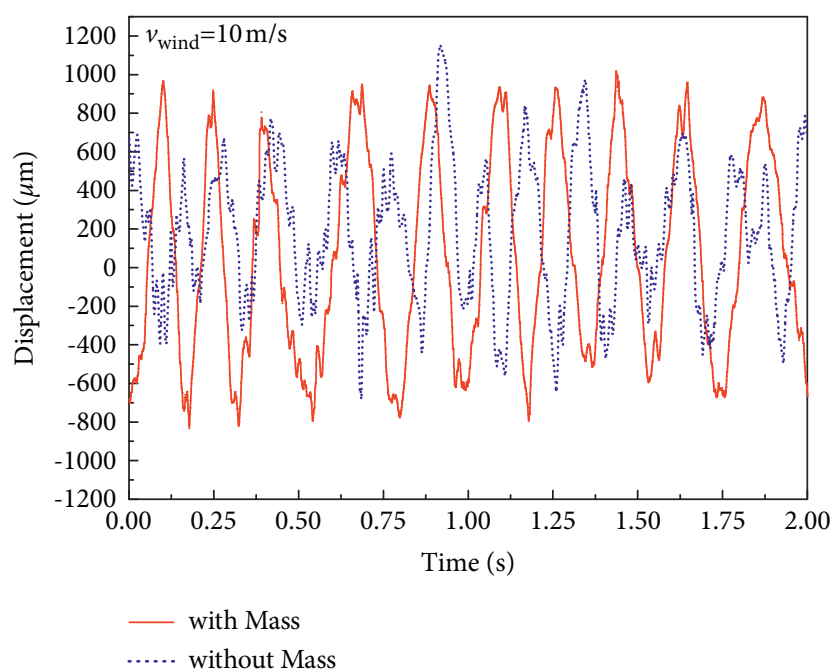

(a)

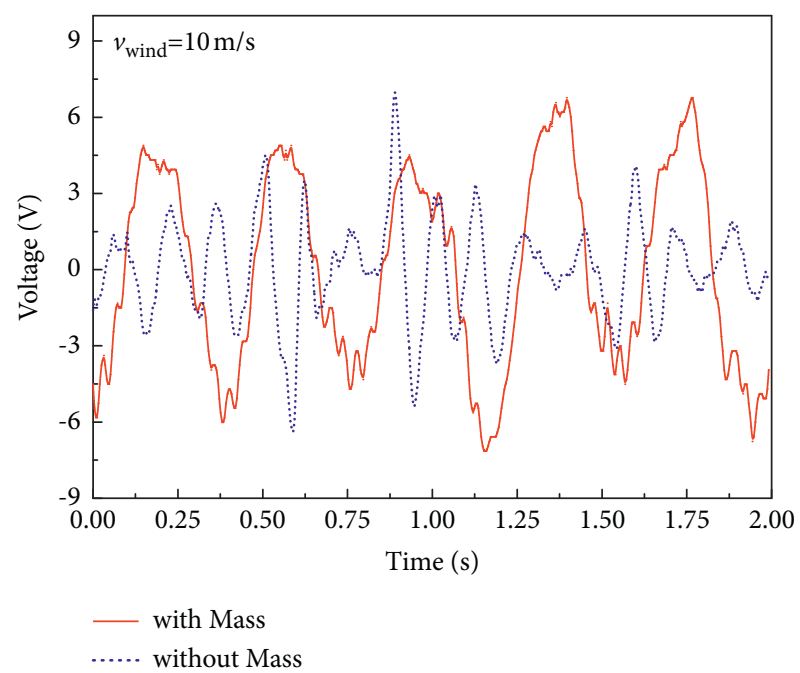

(b)

FIgURE 8: Comparison of (a) vibration displacement amplitude and (b) output AC voltage of the piezoelectric energy harvester before and after adding a metal mass.

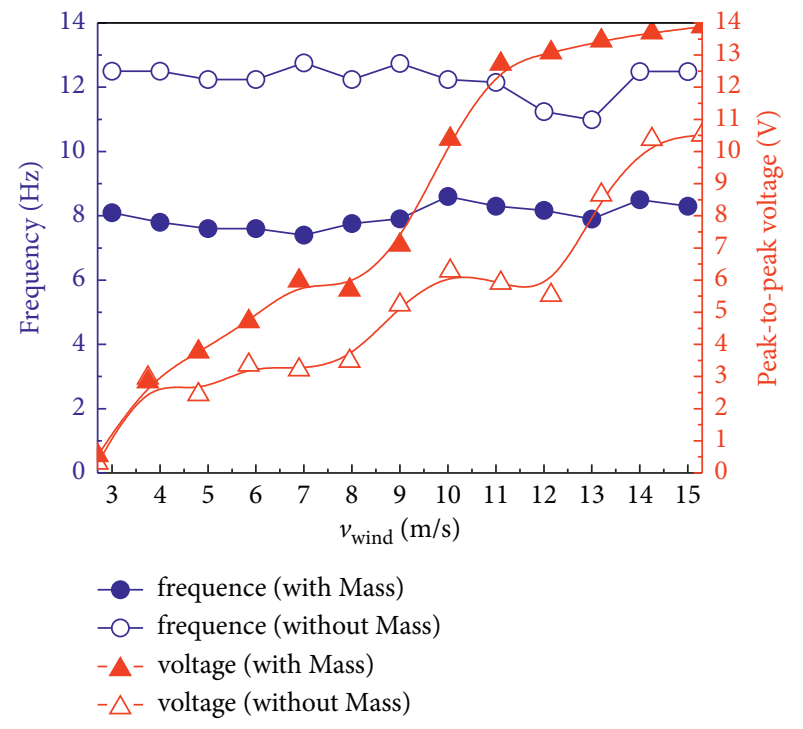

Figure 9: Frequency change and the peak-to-peak voltage value of the piezoelectric energy harvester before and after under different wind speeds.

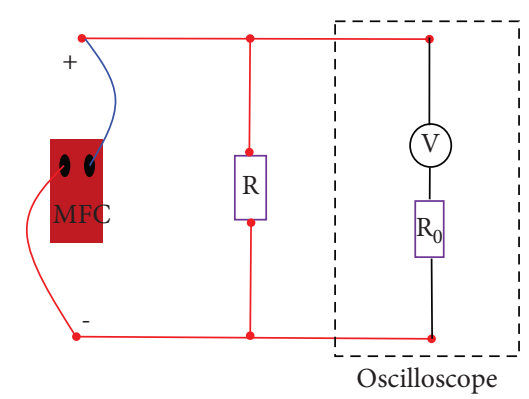

FIGURE 10: Schematic diagram of the closed circuit composed of the flag-swing $\mathrm{PEH}$ and an external impedance.

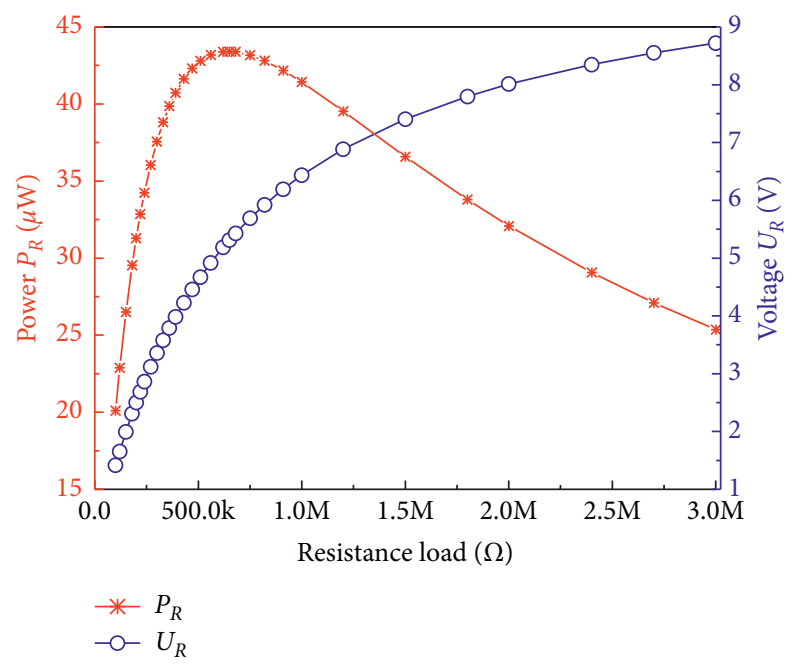

FIGURE 11: Voltage and electric power vary with the resistance changes.

$$
V_{\mathrm{RMS}}=\sqrt{\frac{1}{T} \int_{0}^{T} v(t)^{2} \mathrm{~d} t}
$$

in which $T$ is the period of the AC voltage signal and $v(t)$ represents the instantaneous $\mathrm{AC}$ voltage value output by the energy harvester.

It can be found in Figure 11 that the electric power increases at first and then decreases as the resistance changes. According to equation (1), when the external resistance $R$ is equal to the internal resistance $r$ of the energy harvester, the maximum electric power value is output. That is, when the external resistance $R=650 \mathrm{k} \Omega$, the maximum value of the electric power $P_{R}$ is $43.4 \mu \mathrm{W}$.

Finally, the AC voltage generated by the flag-swing $\mathrm{PEH}$ is measured through a full-wave rectifier circuit (as shown in 


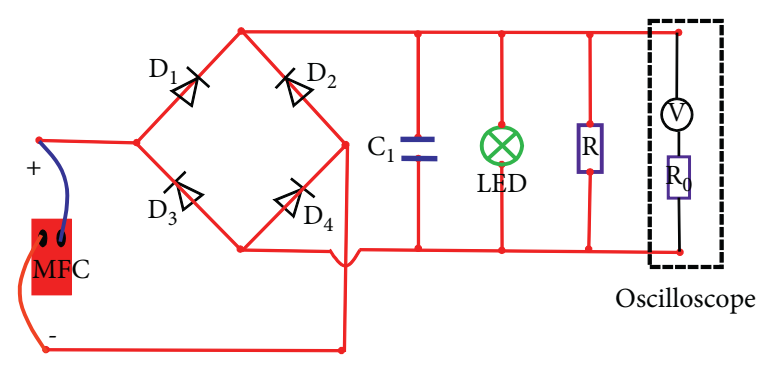

FIGURE 12: Schematic diagram of a full-wave rectifier circuit.

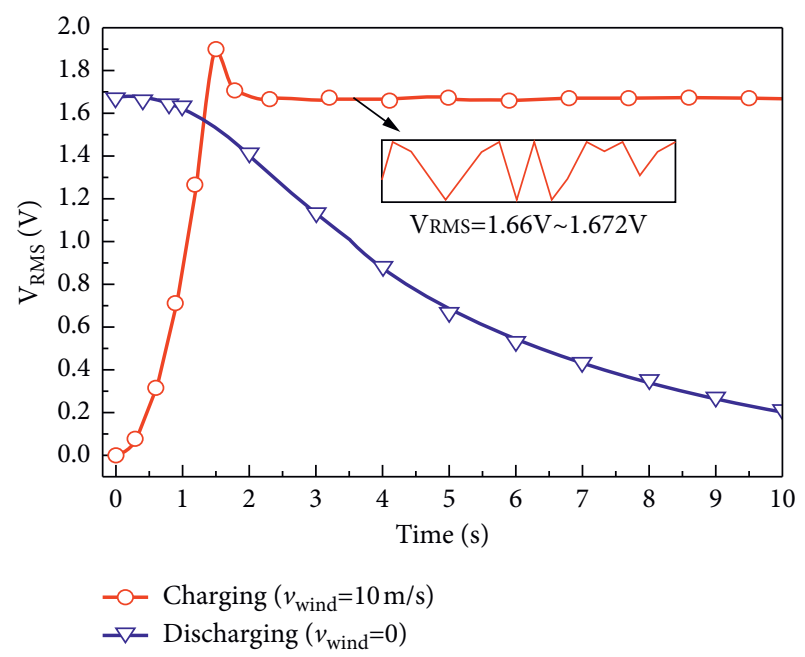

Figure 13: Process of charging and discharging for the circuit load of the harvester after rectification.

Figure 12) to obtain the DC voltage, as shown in Figure 13. In the experiments, after the AC-DC conversion, the $0.1 \mathrm{~W}$ LED lamp could be lit by the harvester, while also obtaining a stable DC voltage of $1.66 \mathrm{~V} \sim 1.72 \mathrm{~V}$, which can meet the power supply requirements of a $1.5 \mathrm{~V}$ chemical battery.

\section{Conclusion}

Inspired by a fluttering ribbon in the wind, this paper designed and investigated the characteristics of a flexible wind-induced flag-swing piezoelectric energy harvester. The harvester can transform wind energy into electric energy based on the piezoelectric effect of piezoelectric materials in order to meet the electrical energy supply needs of low-power electronic devices. The piezoelectric material used in this paper is a flexible P2MFC, which has far more flexibility than the traditional piezoelectric material PZT, allowing it to swing and function in a high deformation environment. Experiments are carried out to investigate the vibration and energy gathering capabilities of the PEH. In addition, as an optimization method, a metal block is placed at the end of the piezoelectric cantilever beam to lower the first-order natural frequency of the $\mathrm{PEH}$ system, allowing it to approach the vibration frequency incited by the wind field. The AC voltage output of the flag-swing $\mathrm{PEH}$ with a metal block has definitely been increased. When the wind speed is $15 \mathrm{~m} / \mathrm{s}$, the energy harvester's AC voltage peak-topeak value can reach $13.88 \mathrm{~V}$ and the maximum electric power in the closed circuit is $P_{\max }=43.4 \mu \mathrm{W}$ with an external resistance of $R$. After the AC-DC conversion, a steady DC voltage of $1.66 \mathrm{~V} \sim 1.72 \mathrm{~V}$ can be achieved, which is sufficient to power a $1.5 \mathrm{~V}$ chemical battery. Simultaneously, the flexible ribbon utilized as the wind-excited vibrator in this article may be freely retracted, reducing the space volume of the $\mathrm{PEH}$ system and allowing the size of the flexible ribbon to be changed based on the needs of the wind farm environment and the power supply device. The suggested energy harvester converts wind energy from a wind farm into electrical energy to power low-power electronic devices, enabling for the generation and usage of green energy to address the issue of insufficient energy in an effective manner.

\section{Data Availability}

Some or all data, models, or code generated or used during the study are available from the corresponding author by request. All data, models, and code generated or used during the study appear in the submitted article.

\section{Conflicts of Interest}

The authors declare that they have no conflicts of interest.

\section{Acknowledgments}

This work was supported by the National Natural Science Foundation of China (Nos. 11772245, 12002256, and 11572235), the Fundamental Research Funds for the Central Universities, XJTU in China (xjh012020014), and the Natural Science Basic Research Plan in Shaanxi Province of China (Program Nos. 2018JC-004 and 2020JQ-011). The author Qun Li gratefully acknowledges the support of K. C. Wong Education Foundation.

\section{References}

[1] Z. Q. Lu, J. Chen, H. Ding, and L. Q. Chen, "Two-span piezoelectric beam energy harvesting," International Journal of Mechanical Sciences, vol. 175, Article ID 105532, 2020.

[2] L. Ding, L. Yang, Z. Yang, L. Zhang, and B. Yang, "Performance improvement of aeroelastic energy harvesters with two symmetrical fin-shaped rods," Journal of Wind Engineering and Industrial Aerodynamics, vol. 196, no. 109, Article ID 104051, 2020.

[3] S. Hassanli, K. Chauhan, M. Zhao, and K. C. S. Kwok, "Application of through-building openings for wind energy harvesting in built environment," Journal of Wind Engineering and Industrial Aerodynamics, vol. 184, pp. 445-455, 2019.

[4] B. Zhu, Y. Huang, and Y. Zhang, "Energy harvesting properties of a flapping wing with an adaptive Gurney flap," Energy, vol. 152, pp. 119-128, Article ID S0360544218305516, 2018.

[5] Y. Yang, L. Zhao, and L. Tang, "Comparative study of tip cross-sections for efficient galloping energy harvesting," Applied Physics Letters, vol. 102, no. 6, 2013.

[6] R. Antons and H. A. Sodano, "A review of power harvesting using piezoelectric materials (2003 2006)," Smart Materials and Structures, vol. 6, no. 3, pp. 1-21, 2007.

[7] Y. Wu, A. Badel, F. Formosa, W. Liu, and A. Agbossou, "Nonlinear vibration energy harvesting device integrating mechanical stoppers used as synchronous mechanical 
switches," Journal of Intelligent Material Systems and Structures, vol. 25, no. 14, pp. 1658-1663, 2014.

[8] L. Sovannarith and N. Hoonchareon, "Stability of the microgrid with wind power generation," in Proceedings of the IEEE International Conference on Sustainable Energy Technologies, Singapore, November 2008.

[9] Y. K. Tan and S. K. Panda, "Optimized wind energy harvesting system using resistance emulator and active rectifier for wireless sensor nodes," IEEE Transactions on Power Electronics, vol. 26, no. 1, pp. 38-50, 2011.

[10] S. G. Li, J. P. Yuan, and H. Lipson, "Ambient wind energy harvesting using cross-flow fluttering," Journal of Applied Physics, vol. 109, no. 2, Article ID 026104, 2020.

[11] C. B. Williams and R. B. Yates, "Analysis of a micro-electric generator for microsystems," Sensors and Actuators A: Physical, vol. 52, no. 1, pp. 8-11, 1996.

[12] E. Bischur, S. Pobering, M. Menacher, and N. Schwesinger, "Piezoelectric energy harvester operating in flowing water," Proceedings of SPIE-The International Society for Optical Engineering 2010, vol. 7643, 2010.

[13] G. W. Taylor, J. R. Burns, S. A. Kammann, W. B. Powers, and T. R. Welsh, "The energy harvesting EEL: a small subsurface ocean/river power generator," IEEE Journal of Oceanic Engineering, vol. 26, no. 4, pp. 539-547, 2001.

[14] S. Priya, C.-T. Chen, D. Fye, and J. Zahnd, "Piezoelectric windmill: a novel solution to remote sensing," Japanese Journal of Applied Physics, vol. 44, no. 3, pp. L104-L107, 2005.

[15] S. Li and H. Lipson, "Vertical-stalk flapping-leaf generator for wind energy harvesting," in Proceedings of the ASME 2009 Conference on Smart Materials, Adaptive Structures and Intelligent Systems, Oxnard, CA, USA, September 2009.

[16] D. S. Clair, A. Bibo, V. R. Sennakesavababu, M. F. Da, and G. Li, "A scalable concept for micro-power generation using flow-induced self-excited oscillations," Applied Physics Letters, vol. 96, no. 14, 2010.

[17] S. Li, J. Yuan, and H. Lipson, "Ambient wind energy harvesting using cross-flow fluttering," Journal of Applied Physics, vol. 109, no. 2, pp. R1-619, 2011.

[18] L. A. Weinstein, M. R. Cacan, P. M. So, and P. K. Wright, "Vortex shedding induced energy harvesting from piezoelectric materials in heating, ventilation and air conditioning flows," Smart Materials and Structures, vol. 21, no. 4, 2012.

[19] J. J. Liu, H. Zuo, W. Xia et al., "Wind energy harvesting using piezoelectric macro fiber composites based on flutter mode," Microelectronic Engineering, vol. 231, no. 4, Article ID 111333, 2020.

[20] R. B. Williams, B. W. Grimsley, D. J. Inman, and W. K. Wilkie, "Manufacturing and mechanics-based characterization of macro fiber composite actuators," in Proceedings of the ASME 2002 International Mechanical Engineering Congress and Exposition, pp. 79-89, New Orleans, LA, USA, November 2002.

[21] M. Peddigari, G. T. Hwang, and J. Ryu, "A comparison study of fatigue behavior of hard and soft piezoelectric single crystal macro-fiber composites for vibration energy harvesting," Sensors, vol. 19, no. 9, 2019.

[22] A. Pandey and A. Arockiarajan, "Performance studies on Macro fiber composite (MFC) under thermal condition using Kirchhoff and Mindlin plate theories," International Journal of Mechanical Sciences, vol. 130, pp. 416-425, 2017. 